\title{
Structure factor of polymers interacting via a short range repulsive potential: application to hairy wormlike micelles
}

\author{
Gladys Massiera * Laurence Ramos, and Christian Ligoure \\ Groupe de Dynamique des Phases Condensées (CNRS-UM2 n $n^{\circ} 581$ ), \\ CC26, Université Montpellier 2, 34095 Montpellier Cedex 5, France. \\ Estelle Pitard \\ Laboratoire de Physique Mathématique et Théorique (CNRS-UM2 no5825), \\ CC50, Université Montpellier 2, 34095 Montpellier Cedex 5, France.
}

(Dated: September 14, 2018)

\begin{abstract}
We use the Random Phase Approximation (RPA) to compute the structure factor, $S(q)$, of a solution of chains interacting through a soft and short range repulsive potential $V$. Above a threshold polymer concentration, whose magnitude is essentially controlled by the range of the potential, $S(q)$ exhibits a peak whose position depends on the concentration. We take advantage of the close analogy between polymers and wormlike micelles and apply our model, using a Gaussian function for $V$, to quantitatively analyze experimental small angle neutron scattering profiles of semi-dilute solutions of hairy wormlike micelles. These samples, which consist in surfactant selfassembled flexible cylinders decorated by amphiphilic copolymer, provide indeed an appropriate experimental model system to study the structure of sterically interacting polymer solutions.
\end{abstract}

*present address: Chemical and Biomolecular Engineering Department, University of Pennsylvania, Towne 311A, Philadelphia, PA 19104, USA. 


\section{INTRODUCTION}

Isotropic solutions of uni-dimensional objects like polymers do not generally show a correlation peak in their structure factor except for very concentrated systems and melts, or charged systems. For polyelectrolytes, the peak originates from strong electrostatic interchains interactions, whereas for neutral polymers the peak is observed at very large scattering vector, on the order of the inverse of the monomer length, and is the signature of a liquid-like order at the monomer length scale, as in simple liquids. Recently we have reported on a new type of living polymer system which also exhibits a structural peak in the scattering function, but with a totally different physical origin [1]. The experimental system is a semidilute solution of hairy wormlike micelles, obtained by adding small amounts of amphiphilic copolymer to a solution of surfactant micelles. The correlation peak, observed even at low concentration of micelles but still in the semi-dilute regime, originates from steric repulsion between the micelles, induced by the copolymer layer which covers them. Despite its short range nature, on the order of the copolymer layer thickness, this interaction is sufficient to generate a correlation peak, as demonstrated by small angle neutron scattering (SANS) experiments. Because surfactant self-assemblies scatter light and neutron much more strongly than polymers, surfactant wormlike micelles have appeared as a convenient model system for the study of the structure of polymer solutions [2]. In particular, charged wormlike micelles have been extensively studied as an alternative system to conventional polyelectrolyte solutions [3, 4, 5]. Similarly, hairy wormlike micelles may be a suitable model system to investigate steric interactions in semi-dilute solutions of polymers.

The Random Phase Approximation (RPA) represents a powerful theoretical tool to predict the structure factor of polymeric systems, provided concentration fluctuations are weak. Introduced a long time ago in the context of simple liquids [6, 7], it has been reformulated explicitly by Edwards in the framework of polymer theory [8], and been applied since to numerous polymer systems, for instance to polyelectrolytes solutions [9, 10, 11, 12] or to microphase separation in block copolymers melts [13]. For concentrated polymer solutions, concentration fluctuations are weak, allowing a perturbative approach for the calculation of correlation functions, starting from the mean field Hamiltonian of the system. A perturbative calculation around the homogeneous equilibrium values of the monomer densities allows in particular the computation of the structure factor. In this paper, we add a Gaussian re- 
pulsive potential to the classical excluded volume interactions between monomers, using a RPA description of polymer solutions (using Edward's formalism) and calculate the structure factor $S(q)$. We show that $S(q)$ may exhibit a broad correlation peak, whose existence and position as a function of polymer concentration, range and magnitude of the Gaussian potential are discussed. The behavior of the theoretical $S(q)$ appears in excellent concordance with the experimental SANS scattering profiles of hairy wormlike micelles. Moreover, we use our model to fit the experimental peak position as a function of the micellar concentration and derive a measurement of the thickness $h$ of the polymer layer covering the micelle. The numerical values of $h$ are found in good agreement with simple theoretical expectations and with other experimental determinations [14].

The paper is organized as follows: Section [II describes the experimental system and recalls the main experimental results reported in Ref. [1] concerning the scattering patterns of semi-dilute solutions of hairy wormlike micelles. In Sec. IIII we describe our model based on the RPA technique and report technical details of the calculations in the appendix. The model, which allows one to predict the structure factor of semi-dilute polymer solutions which interact via a short-range repulsive potential, is applied to our specific experimental system. Finally, in Sec. IV we compare the model to experiments and derive a quantitative evaluation for the thickness of the polymeric layer. We conclude in Sec. $\nabla$ by some remarks concerning the generality of our approach and the validity of the RPA in the framework of semi-dilute solutions of wormlike micelles.

\section{EXPERIMENTS}

\section{A. Experimental system}

Hairy polymers are obtained by adding to solutions of wormlike micelles small amounts of amphiphilic copolymer, whose hydrophobic part adsorbs onto the fluid surfactant cylinders and whose hydrophilic tail remains swollen in water and decorates the micelles. The surfactant micelles are formed by diluting in brine $([\mathrm{NaCl}]=0.5 \mathrm{M})$ a mixture of cetylpyridinium chloride $(\mathrm{CpCl})$ and sodium salicylate $(\mathrm{NaSal})$ at a fixed molar ratio $[\mathrm{NaSal}] /[\mathrm{CpCl}]=0.5$

15]. We use commercially available triblock copolymers (Synperonic F108 and F68, by Serva, used as received) and a diblock copolymer (PC18, synthesized in our laboratory [16]). 
The Synperonic F108 (F68) consists in two identical hydrophilic polyoxyethylene (POE) blocks of 127 (76) monomers each, symmetrically bounded to a central shorter hydrophobic block of polyoxypropylene (PPO) of 48 (29) monomers. The polymer PC18 consists in a $C_{18}$ alkyl chain as hydrophobic part, bounded by an uretane group to a POE block of 113 monomers. The radii of gyration of the hydrophilic blocks are 18.6, 25.8, and $24 \AA$ for F68, F108 and PC18 respectively. We have shown in Ref. [1] that the cylindrical structure of the micelles is maintained upon copolymer addition, with a constant radius of their hydrophobic core $r_{C} \approx 21 \AA$. We define $\phi$ as the surfactant volume fraction and $\alpha$ as the PEO-block to surfactant molar ratio. In our experiments, we vary $\phi$ and $\alpha$ between 2.8 and $40 \%$ and 0 and $4.2 \%$ respectively. The parameter $\alpha$ controls the density of the polymeric layer. The crossover $\alpha^{*}$ between the mushroom regime and the brush regime [17] being estimated to 3\%, $1.5 \%$ and $1.8 \%$ for F68, F108 and PC18 respectively, both regimes are probed in our experiments. On the other hand, in the range of surfactant concentration investigated, the micellar solutions are in the semi-dilute regime.

SANS experiments are performed on the spectrometer PACE at the Laboratoire Léon Brillouin (Saclay, France) and on the D11 beam line at the Institut Laue Langevin (Grenoble, France). We use deuterated water, all other components being hydrogenated. Neutron experiments are thus sensitive to the contrast between the hydrophobic core of the micelles and the aqueous solvent $\left(\mathrm{D}_{2} 0\right)$. In particular, because the hydrophilic POE blocks of the copolymer are always highly swollen in $\mathrm{D}_{2} 0$, the contribution of the copolymer layer to the scattered intensity is negligible compared to the contribution of the hydrophobic core and thus the copolymer layer covering the micelles is not directly probed. In all the experiments, the temperature is fixed at $30^{\circ} \mathrm{C}$.

\section{B. Correlation peak}

In this section, we recall the main experimental results previously reported by us in

Ref.[1]. Figure 10 shows the variation of the scattering profile for samples with constant copolymer density $(\alpha=1 \%)$ but with various surfactant volume fractions $\phi$. The scattering profile is monotonically decreasing at low $\phi$ and above a threshold surfactant volume fraction $\phi_{c}$, a correlation peak is observed at a finite wavevector. The intensity of the peak increases with $\phi$ and its position $q^{*}$ is reported to higher wavevector when $\phi$ increases. Figure 2 a 
shows the variation of the scattering profile at a fixed surfactant volume fraction $\phi=9 \%$ when the copolymer density is increased. A peak emerges above a threshold copolymer molar ratio and becomes more and more pronounced and narrow as $\alpha$ increases. Moreover, the peak position varies only weakly with the copolymer to surfactant ratio. We note that these features are obtained for the three types of copolymer used.

Because of the high ionic strength $(0.5 \mathrm{M})$, electrostatic interactions are screened and are thus not relevant in our experiments. The correlation peak observed experimentally, whose intensity increases with the copolymer over surfactant ratio, originates therefore from the copolymer layer adsorbed onto the micelles. This layer creates a steric short-range repulsion between the micelles, with a range on the order of the copolymer layer thickness. To analyze more quantitatively the scattering profiles, we model the soft short-range copolymerinduced repulsion and use the RPA technique to compute the structure factor of a solution of polymeric chains interacting via a short-range repulsion.

\section{THEORETICAL ANALYSIS}

\section{A. RPA Model}

In a continuous approach, the Hamiltonian $\mathcal{H}(\vec{r}(s))$ of a linear chain of $N$ statistical units in a given configuration $\vec{r}(s)$ reads, in $k_{B} T$ units :

$$
\begin{aligned}
\mathcal{H}(\vec{r}(s))= & \frac{3}{2 a^{2}} \int_{0}^{N} d s\left(\frac{\partial \vec{r}}{\partial s}\right)^{2}+ \\
& +\frac{1}{2} \int_{0}^{N} \int_{0}^{N} d s d s^{\prime} \mathbf{V}\left(\vec{r}(s)-\vec{r}\left(s^{\prime}\right)\right)
\end{aligned}
$$

where $a$ is the monomer size, $\vec{r}(s)$ is the position of the $s^{t h}$-monomer, and $\mathbf{V}\left(\vec{r}-\overrightarrow{r^{\prime}}\right)$ is the monomer-monomer interaction potential. The first term of the right end side of Eq1 represents the entropic contribution of the chain configurations (the "entropic elasticity") and the second term describes the two-body interaction. From this Hamiltonian, we calculate the partition function of the system, and we evaluate the monomer density auto-correlation function, which is directly proportional to the structure factor $S(q)$. The details of these calculations are given in the appendix and can be generalized to a system of $M$ independent chains containing each $N$ monomers [18]. The result is a classical general expression for the structure factor for any given microscopic potential $\mathbf{V}(\vec{r})$ between monomers: 


$$
S^{-1}(\vec{q})=S_{0}^{-1}(\vec{q})+\widetilde{\mathbf{V}}(\vec{q}),
$$

where $\widetilde{\mathbf{V}}(\vec{q})$ is the Fourier transform of $\mathbf{V}(\vec{r})$ and $S_{0}(\vec{q})$ is the structure factor of a Gaussian chain without interaction:

$$
S_{0}(\vec{q})=N V \rho_{0} f\left(\left(q R_{G}\right)^{2}\right),
$$

In Eq 3, $N$ is the number of monomers per chain, $R_{G}$ the radius of gyration of one chain, $V$ the total volume, $\rho_{0}$ the homogeneous equilibrium density of monomers and $f(x)=\frac{2}{x^{2}}\left(e^{-x}+x-1\right)$ the Debye function.

Hence, Eq 2 allows one to calculate the structure factor of semi-dilute solutions of polymers for any given interaction potential between monomers. This equation can be used for hairy wormlike micelles, once a phenomenological potential $\mathbf{V}(\vec{r})$ is given to account for the steric repulsion.

\section{B. Phenomenological repulsive potential}

We assume that the interaction potential between hairy polymers can be considered as the sum of a standard excluded volume potential (polymer/solvent interactions), $v_{0} \delta(\vec{r})$, and an additional repulsive potential, $V_{g}(\vec{r})$, due to the steric layer. The two physical criteria for this steric potential are that $V_{g}(\vec{r})$ should be soft and short range. We thus choose to model it with a Gaussian function, which decreases sufficiently fast to be considered as short range:

$$
V_{g}(\vec{r})=U_{0} \exp \left(-\frac{\mathrm{r}^{2}}{2 \delta^{2}}\right)
$$

We expect $\delta$, the width of the Gaussian, to be on the order of the steric layer thickness. We moreover expect both $\delta$ and the amplitude of the repulsive potential, $U_{0}$, to increase as the amount of copolymer $\alpha$ increases. We note that a Gaussian form for the potential has been recently justified for some soft interacting objects, such as polymer coils [19, 20], flexible

dendrimers 21], or star polymers near the $\theta$-point [22]. A Gaussian shape has the advantage of leading to a simple analytical expression for the structure factor of hairy polymers: 


$$
V S^{-1}(\vec{q})=V S_{0}^{-1}(\vec{q})+v_{0}+U_{0}\left(2 \pi \delta^{2}\right)^{3 / 2} \exp \left(-\frac{(\mathrm{q} \delta)^{2}}{2}\right)
$$

The last two terms of the right end side of Eq 5 represent the interaction part of the structure factor: $v_{0}$ is the excluded volume parameter and the last term is the Fourier transform of $V_{g}(\vec{r})$.

\section{Theoretical structure factor and comparison with experiments}

We take advantage of the close analogy between classical polymers and giant micelles and apply the RPA results to semi-dilute solutions of wormlike micelles. We use Eq $\$ 5$ to compute the structure factor of solutions of hairy micelles and investigate their variation with the micellar concentration and characteristics of the copolymer-induced Gaussian potential.

We define the statistical unit or "monomer" as a slice of micelle of length a equal to $2 l_{P}$, where $l_{P} \simeq 190 \AA$ is the persistence length of the micelle, and of radius $r_{0} \simeq 30 \AA$ [23, 24]. The equilibrium density of monomers, $\rho_{0}$, is then related to the surfactant volume fraction by $\rho_{0}=\phi / 2 \pi r_{0}^{2} l_{P}$. The number of statistical units per chain obeys $N=\frac{a_{0}^{2}}{4 \pi r_{0} l_{P}}\left(\frac{v_{\text {sol. }}}{v_{\text {t.a. }}}\right)^{-1 / 2} \phi^{1 / 2} \exp \left[E / 2 k_{B} T\right][25]$, where $a_{0}=7.2 \AA$ is the surfactant polar head diameter, $E \approx 26 \mathrm{k}_{\mathrm{B}} \mathrm{T}$ is the end-cap energy and $v_{\text {sol. }} \approx 30 \AA^{3}$ and $v_{\text {t.a. }} \approx 595 \AA^{3}$ are the volume of a molecule of solvent and of surfactant respectively. We use this expression for $N$ to calculate the radius of gyration of a chain: $R_{G}=a \sqrt{N} / \sqrt{6}$. The excluded parameter is fixed to $v_{0}=a^{3}$, which corresponds to a polymer in an athermal solvent.

With excluded volume as unique interaction potential, Eq 5 is reduced to the classical expression first derived by Edwards [18] for a polymer chain with excluded volume interactions and the structure factor is a monotonically decreasing function of the $q$ vector. By contrast, in the presence of a Gaussian potential, we show that the structure factor $S(q)$ may have a non-monotonic variation and exhibit a peak at a finite wavevector $q^{*}$. The condition for the existence of a peak can be determined from the analytical expression of the structure factor (Eq 5). This is easily calculated if we note that the derivative of the inverse of the Debye function, $f^{-1}(x)$, tends to $1 / 2$ for large $x$, while it tends to $1 / 3$ when $x$ is small. Assuming that $\left(q R_{G}\right)^{2} \gg 1$, which is always verified in the neighbourhood of the peak, we obtain that the structure factor displays a peak for surfactant volume fraction $\phi$ larger than a threshold value $\phi_{c}$ : 


$$
\phi_{c}=\frac{4 \pi r_{0}^{2} l_{P}^{3}}{3(2 \pi)^{3 / 2}} \frac{1}{\delta^{5} U_{0}}
$$

and the position $q^{*}$ of the peak is given by:

$$
q^{*}=\frac{\sqrt{2}}{\delta} \sqrt{\ln \left(\frac{\phi}{\phi_{c}}\right)}=\frac{\sqrt{2}}{\delta} \sqrt{\ln \left(\frac{3(2 \pi)^{3 / 2}}{4 \pi r_{0}^{2} l_{P}^{3}} \phi \delta^{5} U_{0}\right)}
$$

The critical volume fraction depends on the two parameters characterizing the Gaussian potential, $\delta$ and $U_{0}$. It decreases as either the range or the amplitude of the potential increases, but $\phi_{c}$ is more sensitive to $\delta$ than to $U_{0}$. Note that $\phi_{c}$ diverges if the unique repulsive potential is the excluded volume $\left(\delta=0\right.$ or $\left.U_{0}=0\right)$, consistently with the structure factor being strictly decreasing, as mentioned above.

In order to directly compare the theoretical structure factors with experiments, we first choose fixed values for the parameters of the Gaussian potential, which should correspond to keeping the copolymer over surfactant molar ratio $\alpha$ constant, and we vary the surfactant volume fraction. The theoretical structure factors obtained for $\delta=34 \AA$ and $U_{0}=800 \mathrm{k}_{\mathrm{B}} \mathrm{T}$ are plotted Fig 1b and exhibit features very similar to the experimental scattering profiles (Fig[1). At low $\phi$, the structure factor is a decreasing function of the wavevector. By contrast, for $\phi \simeq 6 \%$, a correlation peak appears, which becomes more and more narrow and whose position is reported to higher wavevector as $\phi$ increases. On the other hand, when the copolymer over surfactant molar ratio $\alpha$ is experimentally varied, the copolymer-induced steric potential changes and thus the two characteristic parameters of the Gaussian potential should change as well. However, there is no clear intuitive argument how to determine the influence of $\alpha$ on $\delta$ and $U_{0}$ separately. To mimic experimental data taken at various $\alpha$, we therefore vary independently $\delta$ and $U_{0}$. The structure factors obtained for $\phi=10 \%$, $U_{0}=800 \mathrm{k}_{\mathrm{B}} \mathrm{T}$ and $\delta$ in the range $5-80 \AA$ are plotted in Fig $2 \mathrm{~b}$, while the structure factors obtained for $\phi=10 \%, \delta=30 \AA$ and $U_{0}$ in the range $200-2500 \mathrm{k}_{\mathrm{B}} \mathrm{T}$ are plotted in Fig,2. Both series display features very similar to the experimental scattering profiles shown in Fig 2 a. For a fixed amplitude $U_{0}$ of the potential, a correlation peak appears for $\delta$ larger than $30 \AA$ and becomes more pronounced as $\delta$ increases. Similarly, increasing $U_{0}$ with fixed $\delta$ leads to the emergence of a peak and to an increase of its intensity. In the two cases, similarly to what is obtained experimentally upon increasing $\alpha$, the parameters $\delta$ and $U_{0}$ have poor influence on the peak position. 
Thus, the theoretical structure factors capture the essential features of the experimental scattering profiles and their evolution with the experimental parameters. However, the comparison between theory and experiment can only be qualitative since theory describes the correlation between the objects and does not take into account the form of the scattering objects, whereas the scattered intensity is experimentally measured. While the relation between scattered intensity, $I(q)$, and structure and form factors, $S(q)$ and $P(q)$, is simple for spherical objects $(I(q)=P(q) S(q))$, it is more complex for semi-dilute solutions of linear and flexible objects. Moreover, the RPA technique usually does not describe correctly the structure factor at very low $q$. These two limitations make it more difficult to fit precisely the experimental scattering profiles, though remarkable agreement with the experimental position of the peak can be found, as shown in the next section.

\section{DISCUSSION}

\section{A. Fit of the peak position}

To analyze more quantitatively our data, we assume that the experimental peak position in the scattered intensity is correctly described by the theoretical structure factor. For surfactant volume fraction above the critical surfactant volume fraction $\phi_{c}$, the peak position $q^{*}$ is given by Eq[7. We use this equation to fit the experimental $\phi$ dependence of $q^{*}$, with $\delta$ and $U_{0}$ as fitting parameters. As shown in fig 3, a very good fit is obtained for the experimental data obtained for hairy micelles decorated with different amounts of copolymer F108 as well as for naked micelles. An equally good agreement is obtained for the two other copolymers (data not shown). The results of the fits are given in Table \for all experimental configurations.

In the case of naked micelles, a correlation peak is detected only at a very large concentration ( $\phi$ above $24 \%$ ) and the $\phi$ dependence of $q^{*}$ can hence be fitted only in a reduced range of concentrations. We find for the fitting parameters $U_{0}=24604 \mathrm{k}_{\mathrm{B}} \mathrm{T}$ and $\delta=13 \AA$, which correspond to a very high and narrow potential. For hairy micelles, the fits always extend over a larger interval of concentrations than that for naked micelles. The values of $U_{0}$ range between 790 and $2770 \mathrm{k}_{\mathrm{B}} \mathrm{T}$ and those of $\delta$ range between 26 and $39 \AA$. Hence, the range of the potential is on the order of the radius of gyration of the copolymer, and is 
always larger than that of naked micelles. It moreover increases from 33.7 to $38.7 \AA$ when $\alpha$ increases from 1 to $3.2 \%$ (for F108 copolymer). In addition, we find $\delta$ smaller for the copolymer F68 than for the copolymers PC18 or F108, as expected, since F68 possesses shorter hydrophilic chains than F108 or PC18. On the other hand, we find that, in the presence of the copolymer layer, the amplitude of the potential is considerably reduced compared to the case of naked micelles. This can be intuitively understood. Indeed, the copolymer layer covering the micelles is presumably very compressible, since this layer is highly swollen by the solvent (the regime of a dry brush is never reached experimentally, $\alpha$ being always comparable to the overlap threshold $\alpha^{*}$ ). This should result in a small value for $U_{0}$, much smaller than for naked micelles for which the dense shell of surfactant polar heads is very little compressible. In fact, for naked micelles, a potential close to a hard core potential is expected, consistently with our results. Although the values of the amplitude $U_{0}$ that we extract from the fits may seem very high, they correspond to very reasonable values for the mean free energy per anchored polymer tail of roughly $15 k_{B} T$.

One could a priori separate the contribution of the surfactant shell from that of the copolymer layer, by replacing the Gaussian potential in Eq2 2 by the sum of two Gaussian functions, one accounting for the surfactant contribution and the other one for the copolymer contribution. The former Gaussian function is expected to be very narrow and high, while the latter is expected wider and lower. We can compute the structure factors in this approach, by taking for the former potential the parameters derived from the fit of the naked micelles and letting free the parameters $\left(\alpha^{\text {copo }}\right.$ and $\left.U_{0}^{\text {copo }}\right)$ for the copolymer contribution. In this case, we find that the essential features of the theoretical structure factors and their evolution with $\phi, \alpha^{\text {copo }}$ and $U_{0}^{\text {copo }}$ remain unchanged. Moreover, the values of $\alpha^{\text {copo }}$ and $U_{0}^{\text {copo }}$, derived from the $\phi$ dependence of $q^{*}$, which should account solely for the copolymer layer, are of the same order of magnitude as the values obtained with one Gaussian function, although $U_{0}^{\text {copo }}$ is slightly smaller than $U_{0}$. This strongly suggests that only the tail of the potential (of energy at most of a few $k_{B} T$ ) is important and that this tail does not vary much with the addition of a high and narrow potential. Moreover it is clear that the concavity of the potential $V_{g}(\vec{r})$ is non-physical for $r<\delta$, limiting the validity of a Gaussian potential to not too short distances.

Finally, we compare the theoretical values of $\phi_{c}$, deduced from the fitting parameters using Eq 6 , to the experimental concentrations. As can be seen in Table ! the experimen- 
tal values show the same variations as the theoretical ones but are systematically smaller. This discrepancy could originate from the fact that what is experimentally measured is the scattered intensity $I$. Peaks of low magnitude in the structure factor may thus be masked in a $I$ vs $q$ plot because of the form factor of the objects, which is a decreasing function of the $q$ vector.

\section{B. Effective thickness of the copolymer layer}

The pair of fitting parameters, $\delta$ and $U_{0}$, allows a determination of an effective thickness of the copolymer layer, $h$. The simple physical criterion we apply is based on the assumption that the micelles enter in contact as soon as their interaction potential overcomes the thermal energy $k_{B} T$. Thus, for a monomer-monomer distance $r=2\left(r_{0}+h\right)$, the Gaussian potential is equal to $1 k_{B} T$ and $V_{g}(r)=U_{0} \exp \left(-r^{2} / 2 \delta^{2}\right)=1$. This criterion leads to a relation between the effective thickness $h$, the naked radius of the micelles $r_{0}$, and the potential parameters $\delta$ and $U_{0}$ :

$$
r_{0}+h=\delta \sqrt{\left(\ln U_{0}\right) / 2}
$$

Using this simple criterion for naked micelles, we obtain $r_{0}=29.2 \AA$ ( $h$ being equal to 0 by definition in this case), a value in striking good agreement with the expected value $\left(r_{0} \simeq 30 \AA[23,24]\right)$. The values of $h$ deduced from the fitting parameters $\delta$ and $U_{0}$ are reported in table $\llbracket$ for hairy micelles and are very close to the radii of gyration of the copolymers. They are also very close, although slightly larger, to the values deduced from two other independent methods [14]. They moreover follow the expected trends: $h$ increases with the amount of copolymer and is larger when the polymer is longer.

\section{Microscopic model for the copolymer-induced repulsive potential}

At this point of the discussion, one can finally raise the question of the microscopic origin of the Gaussian potential used here. With this function for the potential, the model describes correctly the behavior of the scattering profiles and especially the variations of the peak position with $\phi$. It appears nevertheless difficult to draw a precise link between the

microscopic details of the system and the effective mean-field potential. Qualitatively, one can however suggest the following physical picture: the Gaussian potential originates from 


\begin{tabular}{|c||c|c|c|c|c|}
\hline & $\delta(\AA)$ & $U_{0}\left(k_{B} T\right)$ & $h(\AA)$ & theoretical $\phi_{c}$ & experimental $\phi_{c}$ \\
\hline \hline$\alpha=0$ & 13 & 24604 & & $18 \%$ & $25.5 \pm 1.5 \%$ \\
\hline $\mathrm{F} 108, \alpha=1 \%$ & 33.7 & 793 & 31.6 & $4.8 \%$ & $7.3 \pm 0.7 \%$ \\
\hline $\mathrm{F} 108, \alpha=3.2 \%$ & 38.7 & 1007 & 41.9 & $1.9 \%$ & $3 \pm 1 \%$ \\
\hline $\mathrm{F} 68, \alpha=2.1 \%$ & 25.7 & 2768 & 21.2 & $5.3 \%$ & $8 \pm 1 \%$ \\
\hline $\mathrm{PC} 18, \alpha=2.1 \%$ & 33.6 & 1196 & 33.2 & $3.2 \%$ & $4.9 \pm 1.9 \%$ \\
\hline
\end{tabular}

TABLE I: Fitting parameters, range $\delta$ and amplitude $U_{0}$ of the Gaussian potential, effective thickness of the copolymer layer $h$ and theoretical and experimental critical volume fraction above which a correlation peak appears, for samples with different copolymers and different amounts of copolymer.

the copolymer layer covering the wormlike micelles, inducing thereby an additional steric repulsion. Two regimes have to be considered. In the brush regime the micelles are covered with a semi-dilute copolymer layer, while in the mushroom regime the copolymer chains are isolated on the micelles. The interaction is clearly stronger and with a larger range in the brush regime. In order to bring two micelles close to each other, a large energy is needed to compensate the energy cost to compress the copolymer layer below its equilibrium thickness value. This compression energy leads to a strong repulsion which is relevant for short distances $r$ between micelles. This energy can be evaluated from the gap in energy between the equilibrium brush free energy and its value at $r$ in the brush regime, while in the mushroom regime the energy can be evaluated from the energy cost for confining the polymer on distances smaller than its Flory radius. Although the theoretical potentials

calculated with this approach [26] cannot be satisfyingly fit with a Gaussian function, they give numerical values of $h$ in excellent agreement with those deduced from the $q^{*}$ vs $\phi$ fits.

\section{CONCLUSION}

To conclude, we have shown that a RPA approach starting from an Edwards Hamiltonian with a soft Gaussian repulsive potential reproduces well the experimental results obtained for the structure of hairy wormlike micelles, and in particular the variation of the correlation peak with the micellar concentration $\phi$. This model allows one to extract physical parameters 
with very reasonable numerical values.

One can ask whether the RPA is physically justified in the type of systems studied here. Because it essentially neglects strong density fluctuations (see Appendix and [18, 27]), this approximation is a priori best suited for concentrated solutions or melts of polymer. However, this model can nevertheless be applied to less concentrated solutions, provided fluctuations are weak [18]. In Ref.[28], the validity of RPA is discussed in the case of semi-dilute solutions. The authors show that a renormalization of the excluded volume parameter leads to a very good agreement between the RPA and the renormalization group theory. Furthermore, in the case of giant micelles, one can expect to reach a concentrated regime even at relatively low surfactant concentration because of the large persistence length of the micelles. A concentrated regime is indeed attained as soon as the correlation length $\xi$ is on the order of $l_{P}$, which eventually occurs in the range of concentration investigated experimentally. However, in the same range of concentration, several rheology [29] and light scattering [30] experiments indicate that $\xi$ varies as $\phi^{-3 / 4}$, a scaling characteristic of a semidilute solution, in seeming contradiction with the system being in a concentrated regime. This apparent discrepancy underlines how the border between semi-dilute and concentrated solutions is ill defined for wormlike micelles solutions.

Finally, we believe that hairy wormlike micelles solutions provide an original experimental system to illustrate the influence of a soft short range repulsion in isotropic solutions of linear flexible objects. The interaction induced by the copolymer layer is soft but sufficiently strong to induce a correlation peak in the structure factor. The Random Phase Approximation has proven to be helpful in describing the qualitative behavior of the scattered patterns. Such a model may be used in a variety of "hairy linear objects" such as copolymer micelles, or hairy polymers (comblike polymers), for which the interaction is short range. 


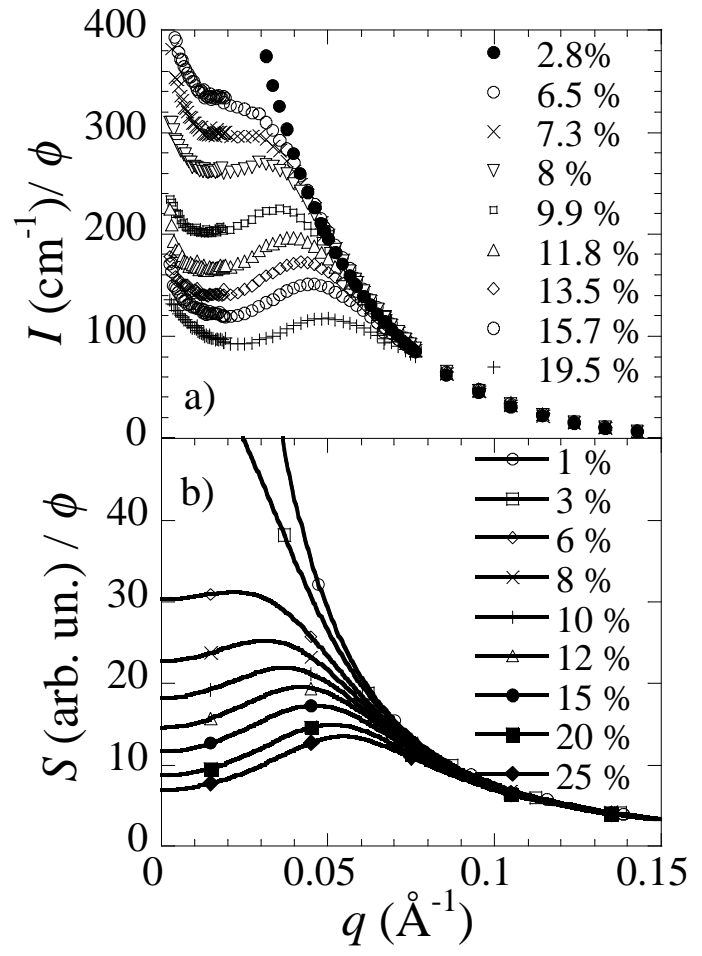

FIG. 1: (a) Experimental scattering profiles and (b) theoretical structure factors, normalized by surfactant volume fraction $\phi$. Curves are labeled by surfactant volume fraction $\phi$. In (a), the copolymer (F108) over surfactant ratio is $\alpha=1 \%$; in (b), the amplitude and range of the Gaussian potential are $U_{0}=800 k_{B} T$ and $\delta=34 \AA$ respectively. 


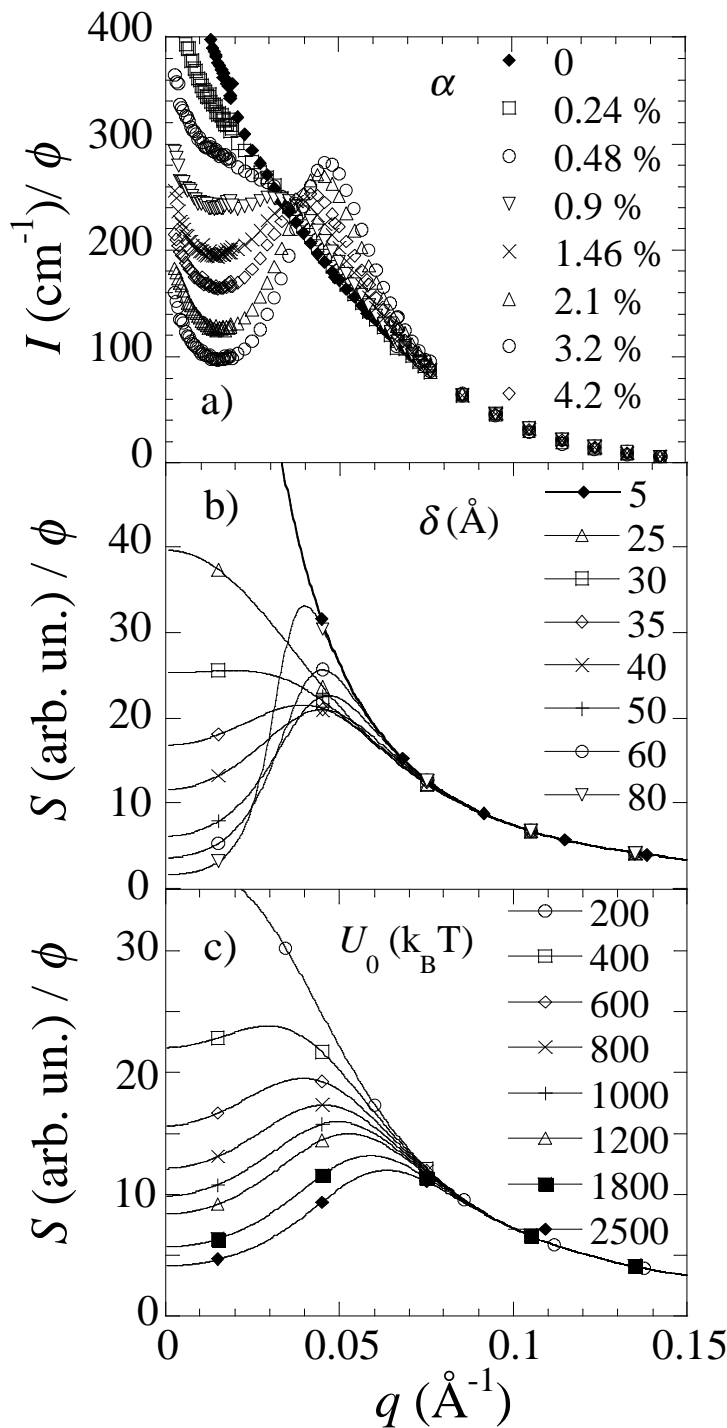

FIG. 2: (a) Experimental scattering profiles and (b, c) theoretical structure factors, normalized by surfactant volume fraction $\phi$. In (a), the surfactant volume fraction is $\phi=9 \%$ and curves are labeled by copolymer (F108) over surfactant ratio $\alpha$. In (b) and (c), the surfactant volume fraction is $\phi=10 \%$. In (b) the amplitude of the potential is $U_{0}=800 k_{B} T$ and curves are labeled by range of the potential $\delta$. In (c), the range of the potential is $\delta=34 \AA$ and curves are labeled by amplitude of the potential $U_{0}$. 


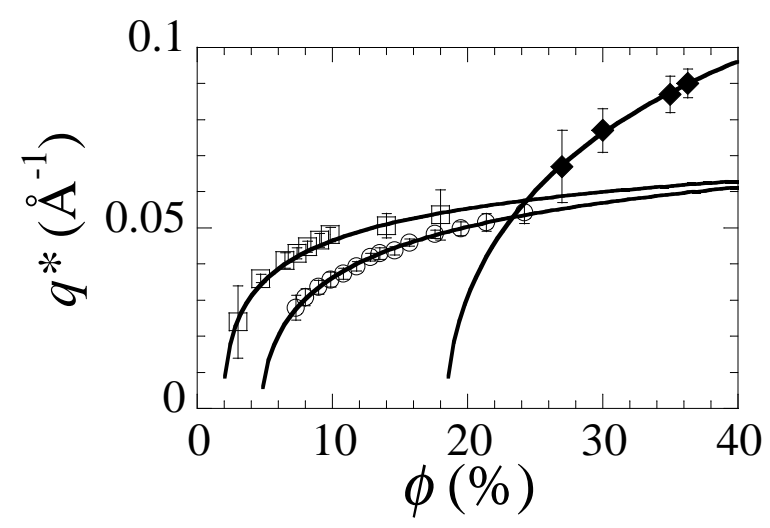

FIG. 3: Variation of the peak position with surfactant volume fraction for samples without copolymer (diamonds), and with a copolymer (F108) over surfactant ratio $\alpha=1 \%$ (empty circles) and $\alpha=3.2 \%$ (empty squares). Symbols are experimental data points and lines are best fits using Eq[7. The fitting parameters are given in table [ 


\section{APPENDIX}

In this appendix, we detail the structure factor calculation for the Random Phase Approximation (RPA).

From the hamiltonian in $k_{B} T$ units (Eq[1),

$$
\mathcal{H}(\vec{r}(s))=\frac{3}{2 a^{2}} \int_{0}^{N} d s\left(\frac{\partial \vec{r}}{\partial s}\right)^{2}+\frac{1}{2} \iint_{0}^{N} d s d s^{\prime} \mathbf{V}\left(\vec{r}(s)-\vec{r}\left(s^{\prime}\right)\right)
$$

we can evaluate the chain partition function $\mathcal{Z}$ as the sum, over all the configurations $\vec{r}(s)$, of the Boltzmann factor:

$$
\mathcal{Z}=\int D \vec{r}(s) \exp [-\mathcal{H}(\overrightarrow{\mathrm{r}}(\mathrm{s}))]
$$

The local monomer density is defined as:

$$
\rho(\vec{r}(s))=\int_{0}^{N} d s \delta(\vec{r}-\vec{r}(s))
$$

From the definition of $\rho(\vec{r}(s))$, we can write the identity:

$$
\int D \rho(\vec{r}) \delta\left(\rho(\vec{r}(s))-\int_{0}^{N} d s \delta(\vec{r}-\vec{r}(s))\right)=1,
$$

which can also be expressed in the Fourier functional space:

$$
\int D \rho(\vec{r}) \int D \hat{\rho}(\vec{r}) \exp \left[\mathrm{i} \int d \vec{r} \widehat{\rho}(\vec{r}) \rho(\vec{r})-i \int d s \widehat{\rho}(\vec{r}(s))\right]=1
$$

By introducing this identity into the partition function, $\mathcal{Z}$ can be expressed as a function of collective variables $\rho(\vec{r})$ and $\widehat{\rho}(\vec{r})$, the local density and its conjugate variable in Fourier space:

$$
\mathcal{Z}=\int D \rho(\vec{r}) D \widehat{\rho}(\vec{r}) \exp [-\mathcal{F}(\{\rho(\overrightarrow{\mathrm{r}}), \widehat{\rho}(\overrightarrow{\mathrm{r}})\})]
$$

with $\mathcal{F}(\{\rho(\overrightarrow{\mathrm{r}}), \hat{\rho}(\overrightarrow{\mathrm{r}})\})=-\ln (\zeta(\{\mathrm{i} \hat{\rho}(\overrightarrow{\mathrm{r}})\}))-\mathrm{i} \int \mathrm{d} \overrightarrow{\mathrm{r}} \hat{\rho}(\overrightarrow{\mathrm{r}}) \rho(\overrightarrow{\mathrm{r}})+\frac{1}{2} \int \mathrm{d} \overrightarrow{\mathrm{r}} \mathrm{d} \overrightarrow{\mathrm{r}^{\prime}} \rho(\overrightarrow{\mathrm{r}}) \mathbf{V}\left(\overrightarrow{\mathrm{r}}-\overrightarrow{\mathrm{r}^{\prime}}\right) \rho\left(\overrightarrow{\mathrm{r}^{\prime}}\right)$

and where $\zeta(\{i \widehat{\rho}(\vec{r})\})$ is a function of $\widehat{\rho}(\vec{r})$ only:

$$
\zeta(\{i \widehat{\rho}(\vec{r})\})=\int D \vec{r}(s) \exp \left[-\frac{3}{2 \mathrm{a}^{2}} \int \mathrm{ds}\left(\frac{\partial \overrightarrow{\mathrm{r}}(\mathrm{s})}{\partial \mathrm{s}}\right)^{2}\right] \exp \left[-\mathrm{i} \int \mathrm{d} \overrightarrow{\mathrm{r}} \hat{\rho}(\overrightarrow{\mathrm{r}}) \quad \mathrm{ds} \delta(\overrightarrow{\mathrm{r}}-\overrightarrow{\mathrm{r}}(\mathrm{s}))\right]
$$


By minimizing the free energy $\mathcal{F}(\{\rho(\vec{r}), \widehat{\rho}(\vec{r})\})$ with respect to both, $\rho(\vec{r})$ and $\widehat{\rho}(\vec{r})$, we obtain $\rho_{0}$ and $\widehat{\rho}_{0}$, the equilibrium homogeneous densities:

$$
\left\{\begin{array} { l } 
{ \frac { \partial \mathcal { F } } { \partial \rho ( \vec { r } ) } | _ { \rho _ { 0 } } = 0 } \\
{ \frac { \partial \mathcal { F } } { \partial i \widehat { \rho } ( \vec { r } ) | _ { i \widehat { \rho _ { 0 } } } = 0 } }
\end{array} \Longleftrightarrow \left\{\begin{array}{l}
\rho_{0}=\frac{N}{V} \\
i \widehat{\rho}_{0}=\rho_{0} W
\end{array}\right.\right.
$$

where $W=\int d \vec{r} \mathbf{V}(\vec{r})$ and $V$ is the total volume.

Assuming fluctuations are weak, we develop a perturbative calculus around the equilibrium homogeneous densities $\left\{\rho_{0}, \widehat{\rho}_{0}\right\}$ :

$$
\left\{\begin{array}{l}
\rho(\vec{r})=\rho_{0}+\delta \rho(\vec{r}) \\
\widehat{\rho}(\vec{r})=\widehat{\rho}_{0}+\delta \widehat{\rho}(\vec{r})
\end{array}\right.
$$

In $\mathcal{Z}$, we only keep constant terms (which contribute to the prefactor $\mathcal{Z}_{0}$ ), and the terms of second order in $\{\delta \rho(\vec{r}), \delta \widehat{\rho}(\vec{r})\}$, the sum of the first order terms being equal to zero. The partition function thus reads:

$$
\begin{aligned}
\mathcal{Z}= & \mathcal{Z}_{0} \int D \delta \rho(\vec{r}) D \delta \hat{\rho}(\vec{r}) \exp \left[\mathrm{i} \int \mathrm{d} \overrightarrow{\mathrm{r}} \delta \rho(\overrightarrow{\mathrm{r}}) \delta \hat{\rho}(\overrightarrow{\mathrm{r}})\right] \exp \left[-\frac{1}{2} \int \mathrm{d} \overrightarrow{\mathrm{r}} \mathrm{d} \overrightarrow{\mathrm{r}^{\prime}} \delta \rho(\overrightarrow{\mathrm{r}}) \mathbf{V}\left(\overrightarrow{\mathrm{r}}-\overrightarrow{\mathrm{r}^{\prime}}\right) \delta \rho\left(\overrightarrow{\mathrm{r}^{\prime}}\right)\right] \\
& \exp \left[-\frac{1}{2} \int \mathrm{d} \overrightarrow{\mathrm{r}} \mathrm{d} \overrightarrow{\mathrm{r}^{\prime}} \delta \hat{\rho}(\overrightarrow{\mathrm{r}}) \frac{\rho_{0}}{\mathrm{~V}} g_{D}\left(\vec{r}-\overrightarrow{r^{\prime}}\right) \delta \hat{\rho}\left(\overrightarrow{r^{\prime}}\right)\right]
\end{aligned}
$$

The function $g_{D}\left(\vec{r}-\overrightarrow{r^{\prime}}\right)$ is the correlation function of a Gaussian chain:

$$
g_{D}\left(\vec{r}-\overrightarrow{r^{\prime}}\right)=\frac{V^{2}}{N} \int D \vec{r}(s) \exp \left[-\frac{3}{2 \mathrm{a}^{2}} \int \mathrm{ds}\left(\frac{\partial \overrightarrow{\mathrm{r}}(\mathrm{s})}{\partial \mathrm{s}}\right)^{2}\right] \int \mathrm{ds} \delta(\overrightarrow{\mathrm{r}}-\overrightarrow{\mathrm{r}}(\mathrm{s})) \int \mathrm{ds}^{\prime} \delta\left(\overrightarrow{\mathrm{r}}^{\prime}-\overrightarrow{\mathrm{r}}\left(\mathrm{s}^{\prime}\right)\right)
$$

Finally, in Fourier space, the partition function reads:

$$
\mathcal{Z}=\mathcal{Z}_{0} \int D \delta \rho(\vec{q}) D \delta \hat{\rho}(\vec{q}) \exp \left[-\frac{1}{2} \int \mathrm{d} \overrightarrow{\mathrm{q}}(\delta \rho(\overrightarrow{\mathrm{q}}), \delta \widehat{\rho}(\overrightarrow{\mathrm{q}})) \mathrm{A}(\overrightarrow{\mathrm{q}})\left(\begin{array}{l}
\delta \rho(-\overrightarrow{\mathrm{q}}) \\
\delta \hat{\rho}(-\overrightarrow{\mathrm{q}})
\end{array}\right)\right]
$$

where the matrix $A(\vec{q})$ is equal to:

$$
A(\vec{q})=V^{2}\left(\begin{array}{cc}
V \widetilde{\mathbf{V}}(\vec{q}) & -i \\
-i & \rho_{0} g_{D}(\vec{q})
\end{array}\right)
$$

where $\widetilde{\mathbf{V}}(\vec{q})$ is the Fourier transform of the interaction potential:

$$
\widetilde{\mathbf{V}}(\vec{q})=\frac{1}{V} \int d \vec{r} e^{-\vec{q} \cdot \vec{r}} \mathbf{V}(\vec{r})
$$


Finally, the Fourier transform of the density fluctuation correlation, which is proportionnal to the structure factor, reads:

$$
S(\vec{q})=V^{2}\langle\delta \rho(\vec{q}) \delta \rho(-\vec{q})\rangle=V^{3}\left[A^{-1}(\vec{q})\right]_{11} .
$$

We thus obtain the simple form of $\mathrm{Eq} 2$ for the structure factor $S(\vec{q})$ :

$$
S^{-1}(\vec{q})=S_{0}^{-1}(\vec{q})+\widetilde{\mathbf{V}}(\vec{q})
$$

where $S_{0}(\vec{q})=N \rho_{0} V f\left(\left(q R_{G}\right)^{2}\right)$, and $f(x)=\frac{2}{x^{2}}\left(e^{-x}+x-1\right)$ is the Debye function.

\section{ACKNOWLEDGMENTS}

We are grateful to R. Aznar for the synthesis of the PC18. Local contacts, L. Auvray at LLB and B. Demé and J. Zipfel at ILL, are acknowledged. E.P thanks Henri Orland for very useful discussions.

[1] G. Massiera, L. Ramos, and C. Ligoure, Langmuir 18, 5687 (2002).

[2] L. M. Walker, Current Opinion in Colloid and Interface Science 6, 451 (2001).

[3] L. J. Magid, J. Phys. Chem. B 102, 4064 (1998).

[4] V. Schmitt and F. Lequeux, J. Phys. II France 5, 193 (1995).

[5] L. Cannavacciuolo, J. S. Pedersen, and P. Schurtenberger, Langmuir 18, 2922 (2002).

[6] H. C. Andersen and D. Chandler, J. Chem. Phys 53, 547 (1970).

[7] J.-P. Hansen and I. R. McDonald, Theory of simple liquids (Academic Press, London, 1990).

[8] S. F. Edwards, Proc. Phys. Soc 88, 265 (1966).

[9] J.-L. Barrat and J.-F. Joanny, Adv. Chem. Phys. XCICV, 1 (1996).

[10] V. Y. Borue and I. Y. Erukhimovich, Macromolecules 21, 3240 (1988).

[11] J.-F. Joanny and L. Leibler, J. Phys. France 51, 545 (1990).

[12] M. Castelnovo and J.-F. Joanny, Eur. Phys. J. E 6, 377 (2001).

[13] L. Leibler, Macromolecules 13, 1602 (1980).

[14] G. Massiera, L. Ramos, E. Pitard, and C. Ligoure, J. Phys.: Condens. Matter 15, S225 (2003).

[15] H. Rehage and H. Hoffmann, J. Phys. Chem. 92, 4712 (1988). 
[16] P. Hartmann, M. Viguier, A. Collet, and D. Calvet, J. Fluorine Chem. 95, 145 (1999).

[17] P.-G. De Gennes, Macromolecules 13, 1069 (1980).

[18] M. Doi and S. F. Edwards, The theory of polymer dynamics, International series of monographs on physics 73 (Clarendon Press, Oxford, 1986), oxford science publications ed.

[19] A. A. Louis, P. G. Bolhuis, J.-P. Hansen, and E. J. Meijer, Phys. Rev. Lett. 85, 2522 (2000).

[20] P. G. Bolhuis, A. A. Louis, J.-P. Hansen, and E. J. Meijer, J. Chem. Phys. 114, 4296 (2001).

[21] C. N. Likos, S. Rosenfeldt, N. Dingeouts, M. Ballauff, P. Lindner, N. Werner, and F. Vogtle, J. Chem. Phys. 117, 1869 (2002).

[22] H. Graf and H. Löween, Phys. Rev. E 57, 5744 (1998).

[23] J. Marignan, J. Appell, P. Bassereau, G. Porte, and R. May, J. Phys. France 50, 2553 (1989).

[24] J. Appell and J. Marignan, J. Phys. France II 1, 1447 (1991).

[25] M. E. Cates and S. J. Candau, J. Phys. Condens. Matter 2, 6869 (1990).

[26] G. Massiera, Thesis, Université de Montpellier II (2002).

[27] P.-G. De Gennes, Scaling concepts in polymer physics (Cornell University, New York, 1979).

[28] M. Müller, K. Binder, and L. Schäfer, Macromolecules 33, 4568 (2000).

[29] G. Massiera, L. Ramos, and C. Ligoure, Europhys. Lett. 57, 127 (2002).

[30] J.-F. Berret, J. Appell, and G. Porte, Langmuir 9, 2851 (1993). 